\title{
Unique pharmacist competency program at community-based, teaching hospitals
}

\author{
Tu T. Tran'1, Shareen El-I biary ${ }^{2}$, Robert Tindula ${ }^{3}$, Neepa Rai ${ }^{3}$, Austin Nguyen ${ }^{3}$, Jennifer Le ${ }^{3,4}$ \\ 1. Huntington Hospital, Pasadena, CA, USA. 2. Midwestern University College of Pharmacy, Department of Pharmacy \\ Practice, Glendale, AZ, USA. 3. Long Beach Memorial Hospital, Department of Inpatient Pharmacy, Long Beach, CA, USA. \\ 4. University of California, San Diego, Skaggs School of Pharmacy and Pharmaceutical Sciences, La Jolla, CA, USA
}

Correspondence: Tu T. Tran. Address: PGY-1 Pharmacy Practice Resident 100 W. California Blvd. Pasadena, CA 91105, USA. E-mail: tu.tran@huntingtonhospital.com

Received: March 7, 2013

Accepted: March 19, 2013

Online Published: April 9, 2013

DOI : $10.5430 /$ jha.v2n3p119

URL: http://dx.doi.org/10.5430/jha.v2n3p119

\section{Abstract}

Background: The pharmacy profession continues to evolve and shape itself with increasing complexity. With this escalating complexity, pharmacist clinical competency needs to be addressed within each healthcare practice setting. The objectives of this study were to describe a unique pharmacist competency program and evaluate its satisfaction at two community hospitals.

Methods: Long Beach Memorial and Miller Children's Hospital of Long Beach are tertiary community hospitals with 308 total beds for Millers Children's Hospital and 462 total beds for Long Beach Memorial. A unique and intensive pharmacist competency program has been established at these hospitals for over 20 years. The content of this program was assessed and a survey was conducted in March 2011 to ascertain pharmacist satisfaction.

Results: The unique pharmacist competency program was structured in the form of age-related, hospital-wide and unit specific modules, pharmacy-regulated therapies (PRT), and a Pharmacy Skills Day that provide updates on PRT and other pharmacy-related topics. Forty-two of $61(69 \%)$ pharmacists responded to the survey. Mean age of pharmacists was $38.8 \pm 11.5$ years, $36 \%$ were male, $86 \%$ completed residency training, and $12 \%$ were board-certified pharmacotherapy specialists. Over $80 \%$ of pharmacists agreed that the program was informative and supportive of their daily patient care activities. Although the program was well-received by the pharmacists, there were facets of the program that needed improvement, including resources for continuing education opportunities and additional modules for competency.

Conclusion: A unique pharmacist competency program at two community hospitals was described. The program was well-received by the pharmacists, and, more importantly, ensured continuous professional development in pharmacy practice.

\section{Key words}

Education, Pharmacist, Competency, Pharmacy skills days, Hospital

\section{I ntroduction}

The advent of new drugs and practice guidelines to enhance medication use continues to augment the complexity of clinical pharmacy practice. As a result, acquiring new knowledge and skills is essential for professional competency. New 
knowledge and skills must first be learned, and then maintained in order to apply directly to patient care with the ultimate goal of optimizing medication use outcomes. Programs designed to guide professional competency should encompass these facets of learning and, most importantly, translate into clinical practice. According to the International Pharmaceutical Federation, it is "the responsibility of individual pharmacists for systematic maintenance, development and broadening of knowledge, skills and attitudes, to ensure continuing competency as a professional throughout their careers" ${ }^{[1]}$.

Continuing education (CE) programs have traditionally been the primary vehicle for pharmacists to maintain professional competency ${ }^{[2]}$. While CE programs provide a pathway to obtain new knowledge, they do not ensure clinical application and thereby are not always translated into clinical practice to reinforce effective medication outcomes. With these clinical limitations, another approach to complement $\mathrm{CE}$ programs is essential to ensure pharmacists receive adequate training to practice at their highest potential. One such mechanism is continuous professional development (CPD), which allows for ongoing, interactive education with assessment of educational performance and needs ${ }^{[2]}$. It was found that in one study comparing CPD vs. traditional CE programs, pharmacists who adopted the CPD approach are more likely to report that various aspects of their learning behaviors improved as a result of education activities then pharmacists who participated in traditional $\mathrm{CE}^{[3]}$.

Continuing professional development is a framework that has been established in conjunction with $\mathrm{CE}$ to provide the competency needed by a pharmacist to function at a high level. In one article, CPD was defined as an ongoing, self-directed, structured, outcomes-focused cycle of learning and personal development. It is an approach to life-long learning that meets the needs of patients and delivers positive health outcomes ${ }^{[2]}$. It is critical for a pharmacist to not only attain technical competencies but also to be able to apply what he or she has learned in their daily practice in order to enhance patient outcomes. According to a survey regarding attitudes towards CPD, workplace learning is a pivotal yet under-emphasized component of CPD and that peer-support is vital in adopting a CPD paradigm ${ }^{[3]}$.

The inpatient pharmacy departments at Long Beach Memorial Hospital (LBM) and Miller Children's Hospital of Long Beach (MCHLB), two community-based, teaching hospitals serving adults and pediatrics, respectively, established a program for CPD to maintain pharmacists' competencies three years ago. This pharmacist competency program had three unique features: (1) self-directed requiring self-motivation and pharmacist willingness to complete modules; (2) based on needs within pharmacy practice that were identified by the clinical coordinators and department managers; and (3) associated with outcomes that enhanced a pharmacist's ability to perform daily clinical and distributive operations. The objectives of our study were to describe our unique pharmacist competency program that incorporated CPD and to assess program satisfaction.

\section{Methods}

Both LBM and MCHLB have 770-combined licensed beds and over 60 pharmacists. LBM consists of 462 beds with 61 ICU beds while MCHLB consists of 308 beds with 20 PICU and 94 Center for Women beds. Interviews were conducted with the director and coordinators of clinical pharmacy at LBM and MCHLB in August 2010 to obtain a description of the program. Along with these interviews, a review of guidelines and/or protocols was conducted to identify the types of activities used for the pharmacist competency program. Approval for this research was obtained from the Institution Review Boards at Memorial Health Systems that included LBM and MCH and Midwestern University College of Pharmacy at Glendale.

To assess satisfaction of the competency program, we electronically surveyed practicing pharmacists at both LBM and MCHLB in March 2011 using Survey Monkey ${ }^{\mathrm{TM}}$. A needs assessment for program enhancement was also evaluated using this survey. An initial email was sent to all pharmacists for voluntary, de-identified, and confidential participation. A 
second email reminder was sent one week prior to the deadline. To enhance the response rate, paper copies of the survey were also delivered to pharmacists' mail-slots. They were asked to complete the survey once, either electronic or paper.

The results of the survey were compiled by one investigator who was not an employee at LBM or MCHLB to ensure complete confidentiality. The data were analyzed using SPSS GradPack 19 (SPSS Inc., Chicago, IL) to determine the percent affected for nominal or ordinal variables, and measures of central tendencies for continuous variables. Study subjects were divided into two groups when necessary and further analyzed using appropriate statistical methods [i.e., $\chi^{2}$ tests or Fisher's exact test for cross-tabulations with nominal variables, independent samples Student t-test for continuous variables, and Wilcoxon rank-sum test for ordinal variables (e.g., comparing if the pharmacist competency program was informative or supportive between age groups)]. All statistical tests were performed with two-tailed analyses. Statistical differences between groups were considered significant when $p$-values were $\leqslant 0.05$.

\section{Results}

Description of Pharmacist Competency Program

The pharmacist competency program at LBM and MCHLB is maintained by the pharmacy director and two part-time clinical coordinators, one for each hospital. It incorporates three major components:

a) Initial qualification process for pharmacists in specific pharmacist-regulated therapies;

b) Hospital-wide competencies, such as safety, infection control, and caring for patients in different age groups; and

c) Pharmacy Skills Day that update clinical and distributive functions of pharmacy practice.

Table 1. Pharmacy-Regulated Therapies

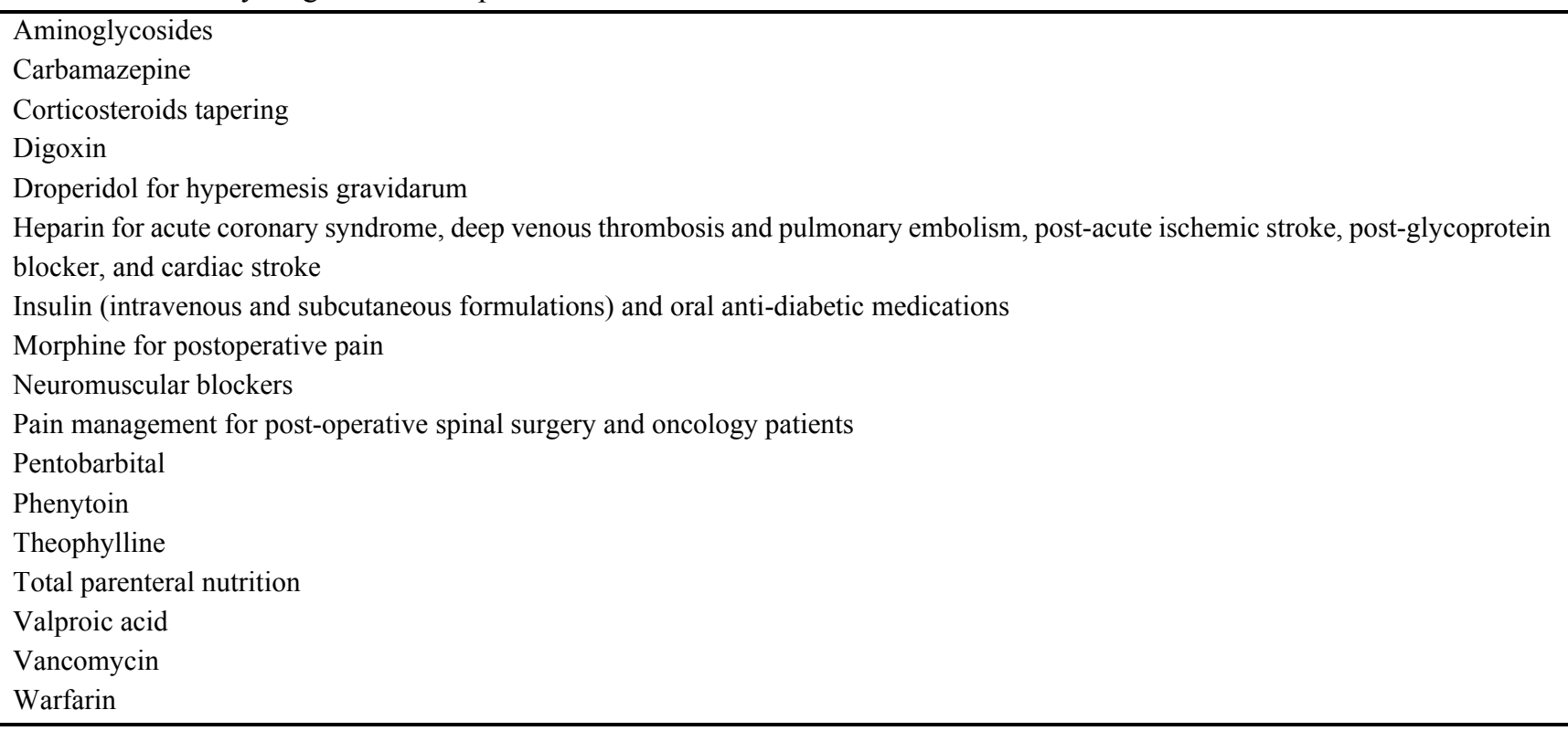

The initial qualification process for the pharmacist competency program utilizes modular-based tools to educate and establish clinical proficiency. These modules consist of pharmacy-regulated therapies that were developed for pharmacy practice at LBM and MCHLB over two decades ago. Pharmacy-regulated therapies are essentially guidelines for therapeutic monitoring of certain drugs, including aminoglycosides, carbamazepine, heparin, warfarin, vancomycin, and 
phenytoin (Table 1). In order to staff a clinical shift, a pharmacist must be qualified in pharmacy-regulated therapies. The type of pharmacy-regulated therapies needed for qualification depends on the area or unit of practice. While all pharmacists must be qualified in vancomycin and aminoglycosides pharmacy-regulated therapies (primarily due to the extensive use of these therapies at LBM and MCHLB), pharmacists working in the labor and delivery would also need qualification in initiating and maintaining unit-specific therapies such as insulin for gestational diabetes and droperidol for hyperemesis gravidarum. This initial qualification in pharmacy-regulated therapies requires successful completion of a written with a score of $80 \%$ or higher. For some pharmacy-regulated therapies, the pharmacist must also present 10 patient cases (five where the pharmacist initiated the therapy and five where the pharmacist made a change to the therapy) to the guideline coordinator to ensure competency in that area. The guideline coordinator is the person in charge of all competency-based training for the pharmacists regarding the specific pharmacist-regulated therapy and provides the annual updates on any changes that become part of Pharmacy Skills Day.

Table 2. Modules for Pharmacy Skills Day 2012

\begin{tabular}{|c|c|c|}
\hline Module No. & Module name & Required for \\
\hline $1 \mathrm{~A}$ & LBM Epic workflow and distribution module & $\begin{array}{l}\text { All adult clinical pharmacists, night pharmacists and support } \\
\text { staff }\end{array}$ \\
\hline $1 \mathrm{P}$ & MCHLB Epic workflow and distribution module & $\begin{array}{l}\text { All pediatric clinical pharmacists, pediatric night pharmacists, } \\
\text { and pediatric support staff }\end{array}$ \\
\hline 2 & Pharmacy One-Source quantify user's guide & $\begin{array}{l}\text { All pharmacists - this is the phramacy's intervention tracking } \\
\text { program }\end{array}$ \\
\hline 3 & $\begin{array}{l}\text { Policies related to pharmacy-regulated therapies } \\
\text { and guideline qualifications }\end{array}$ & All pharmacists \\
\hline 4 & Community-acquired pneumonia measures & All pharmacists \\
\hline 5 & $\begin{array}{l}\text { National Patient Safety Goal for anticoagulation } \\
\text { monitoring }\end{array}$ & All pharmacists \\
\hline 6 & Alteplase preparation in stroke (LBM) & $\begin{array}{l}\text { All adult clinical pharmacists, night pharmacists and support } \\
\text { staff }\end{array}$ \\
\hline 7 & Adding the anticoagulant teaching plan & $\begin{array}{l}\text { All pharmacists - to improve patient teaching documentation } \\
\text { in the medical record }\end{array}$ \\
\hline 8 & Policies and procedure updates and info & All pharmacists \\
\hline 9 & Blood factors (LBM) & $\begin{array}{l}\text { All adult clinical pharmacists, night pharmacists and support } \\
\text { staff }\end{array}$ \\
\hline 10 & $\begin{array}{l}\text { Recombinant factor VIIIa in cardiac surgery } \\
\text { (LBM) }\end{array}$ & $\begin{array}{l}\text { All adult clinical pharmacists, night pharmacists, and support } \\
\text { staff }\end{array}$ \\
\hline 11 & Surgical Care Improvement Plan for pharmacists & All pharmacists \\
\hline
\end{tabular}

LBM = Long Beach Memorial Hospital; MCHLB = Miller Children's Hospital of Long Beach

In conjunction with the pharmacy-regulated therapies, all pharmacists are required to complete hospital-wide modules that comprise of infection control and safety modules and age-specific modules for geriatrics and pediatrics. A unique and innovative idea for maintaining competency developed by the Director of Inpatient Services at LBM is Pharmacy Skills Day. The goal of the Pharmacy Skills Day is to educate as well as maintain pharmacist competency in both drug distribution and clinical practice throughout all areas of the hospitals. This concept was extrapolated from the skills days in the Nursing Department when nurses complete modules related to their scope of practice. Due to the decentralized nature of the pharmacy services at LBM/MCHLB, a packet of information is distributed to all pharmacists for Pharmacy Skills Day. This packet contains different modules that the pharmacist needs to complete based on their area of practice (Table 2). The pharmacist must complete the modules within 4 to 6 weeks and submit the answer sheet during a practical session. Multiple practical sessions are held to accommodate all pharmacist staff, and led by the pharmacy supervisors to 
clarify responses to questions and address concerns. Different topics for the modules are created each year to accommodate updates and/or changes in clinical and distributive practices of pharmacy at LBM/MCHLB.

\section{Survey}

A total of 61 pharmacists were surveyed and 42 pharmacists completed the survey, resulting in a response rate of $69 \%$. The demographics of the pharmacist respondents are shown in Table 3 . Two-thirds of the respondents were $<40$ years old. All pharmacists are certified in basic life support with a median of 12 years since graduation and 6 years of pharmacy practice at LBM/MCHLB. While $86 \%$ of respondents were residency-trained, only $12 \%$ were Board-Certified Pharmacotherapy Specialists (BCPS). Compared to those 35 years or older, those younger than 35 years were more likely BCPS-certified $(13.2 \%$ vs. $39.5 \%, p=0.048)$.

Table 3. Demographic characteristics

\begin{tabular}{lc}
\hline Characteristic & Value $\mathbf{( N = 4 2 )}$ \\
\hline Median age (interquartile range), years & $34(30-51)$ \\
Male gender & $15(36)$ \\
Residency-trained & $36(86)$ \\
Board-Certified Pharmacotherapy Specialist (BCPS) & $5(12)$ \\
Median years since graduation & $12(4-30$ years $)$ \\
Median years worked at study hospitals & $6(4-31$ years $)$ \\
\hline
\end{tabular}

Most pharmacists (88\%) strongly agreed or agreed that pharmacist competency program was informative (Figure 1). None of the respondents disagreed to this survey question. Similarly, $87 \%$ of pharmacists strongly agreed or agreed that the program was helpful in supporting their daily responsibilities. Recommendations to improve the pharmacist competency program were to provide additional resources in the form of CEs and modules to develop competency and time to complete such programs (Table 4).

Table 4. Recommended improvements for pharmacist competency program $(\mathrm{N}=42)^{\mathrm{a}}$

\begin{tabular}{ll}
\hline Type of improvement & No. (\%) \\
\hline Need for more educational resources & $33(79)$ \\
Improve access to program & $11(26)$ \\
Decrease the amount of material in each module & $9(21)$ \\
Extend time to complete program & $8(20)$ \\
\hline
\end{tabular}

${ }^{a}$ Number (\%) unless otherwise stated.

Figure 1. Satisfaction of the pharmacist competency program $(\mathrm{N}=42)^{\mathrm{a}}$

${ }^{a}$ Scale from 1 to 5 , with 5 representing strongly agree.

Informative: Mean $\pm \mathrm{SD}=4.12 \pm 0.59$

Supportive: Mean $\pm \mathrm{SD}=4.02 \pm 0.56$

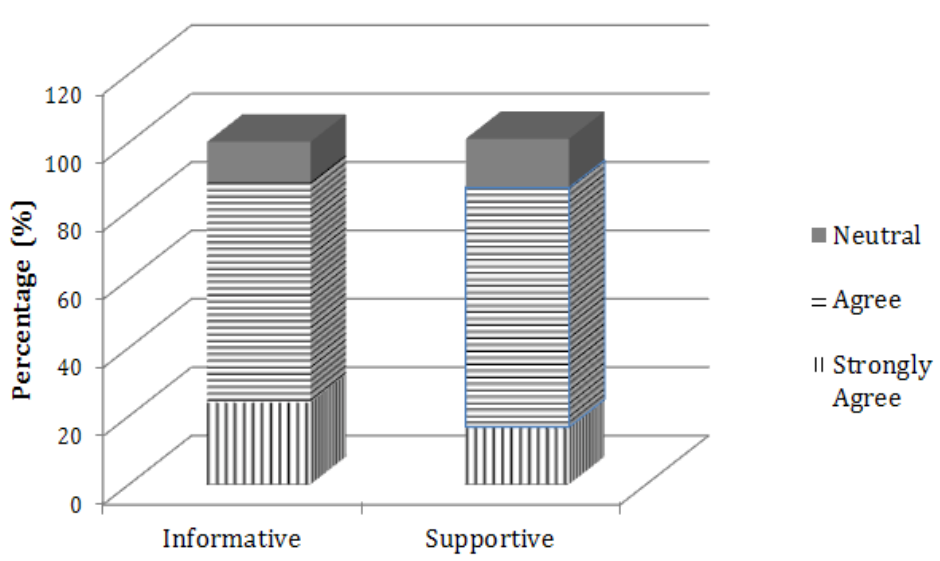




\section{Discussion}

This report describes a unique pharmacist competency program that incorporates the CPD model at two community hospitals. This survey demonstrated that all pharmacists found the competency program informative and supportive of their daily patient care activities. Furthermore, over $70 \%$ of the pharmacist believed the program's pace and access (i.e., modules allowed for schedule flexibility and the direct meeting during Pharmacy Skills Day accommodated different work shifts) were adequate. Notably, none of the pharmacists disagreed to these aspects of the program. The need for additional resources to enhance the competency program was identified.

Due to the number of pharmacy-regulated therapies, the need for a residency is essential. The pharmacy-regulated therapies include drugs with narrow therapeutic indexes so pharmacists are best at implementing and monitoring these therapies. Over $80 \%$ of the pharmacists at LBM/MCHLB, while only $13 \%$ of the staff was BCPS certified. Most agreed that there is a need for continues education to enhance knowledge and skills that support daily activities.

The age of pharmacist at LBM/MCHLB ranges from 30-51 years old with individuals needing different methods to maintain knowledge. Along with the traditional forms of education such as CE programs, the competency program tailors towards specific functional needs, clinical skills (Table 1) and distribution (Table 2) in the forms of Pharmacy Skills Day. The need for such a competency program is essential because many of the pharmacists require a means of continuing education that meets both clinical and functional needs of a particular hospital.

The use of modular based tools with Pharmacy Skills Day provides opportunities for pharmacists to translate their knowledge into clinical practice and also it provides the opportunities for the clinical coordinator to assess the need for further education opportunities to optimize patient care. The uniqueness of the Pharmacy Skills Day provides the opportunity for clinical questions that arise on a daily basis to be answered in an environment that stimulates debate and discussion. Qualification is not presumed with the completion of the modules but more importantly with an exam to ensure competency has been achieved. This unique program provides an opportunity to ask questions as well as ensures that each individual pharmacist achieves and maintains a level of competence for direct patient care activities.

Under the doctrine of corporate liability, the hospital has a legal obligation to select and retain only competent staff members. In addition, the hospital must continuously evaluate all individuals with designated patient care privileges ${ }^{[5-7]}$. As such, the hospital must develop and implement processes for assessing competencies. The pharmacist competency program at LBM/MCHLB provides a model that selects and maintains pharmacists who are qualified to perform their daily patient care activities. Specifically, Pharmacy Skills Day, along with pharmacist regulated therapies, and hospital-wide modules, provides the hospital a method to annually evaluate current knowledge and skills. This continuous competency program may potentially prevent and reduce medication errors.

There were several limitations to our study. First, the study evaluated two community-based hospitals with strong clinical pharmacy services. The pharmacist competency program is maintained by a pharmacy director and two part-time clinical coordinators. The feasibility of initiating and maintaining such a program would be challenging for hospitals without this infrastructure.

On the other hand, this program is unique in the sense that it provides the educational opportunities that maintain competencies to ensure positive patient outcomes. This study was meant to describe a unique program that is implemented at two community hospitals and convey the drawbacks and benefits of such programs.

\section{Conclusions}

This report provides a descriptive analysis of the current pharmacist competency program and attitudes about these programs at two local community hospitals. The responses by the 2010 survey at LBM and MCHLB suggest that a 
majority of the pharmacists were receptive to the competency program at LBM/MCHLB and that most found it beneficial in supporting their daily responsibilities and activities. Pharmacists are accepting of programs and modules such as Pharmacy Skills Day and other pharmacy related activities to develop and maintain competencies. Continuous professional development should not be seen as a replacement for our current CE model but rather a supplement to ensure that a pharmacist is maintaining his or her competency throughout their career. The success of this type of competency program may be implemented and beneficial for other community-based institutions to enhance and meet the demand for pharmacists' competencies.

\section{Conflict of interests}

All authors in this manuscript declare that there are no actual or potential conflicts of interest. There are no financial interests of the authors or members of their immediate families in products or service discussed in the manuscript.

\section{References}

[1] International Pharmaceutical Federation. FIP Statement of progessional standards continuing professional development. 2002.

[2] Rouse MJ. Continuing professional development in pharmacy. Am J Health-Syst Pharm. 2004; 61: 2069-2076. PMid:15509129

[3] McConell KJ, Delate T, Newlon CL. Impact of continuing professional development versus traditional continuing pharmacy education on learning behaviors. J Am Pharm Assoc. 2012 Nov-Dec; 52(6): 745-52.

[4] Austin Z, Marini Anthony, Glover NM, et.al. Continuous Professional Development: A qualitative Study of Pharmacists' Attitudes, Behaviors, and Preferences in Ontario, Canada. Am J Pharm Educ. 2005; 69(1): Article 4.

[5] Merrigan D. Internal approach to competency-based credentialing for hospital clinical pharmacists. Am J Health-Syst Pharm. 2002; 59: 552-558. PMid: 11908250

[6] Bellanger RA, Shank TC. Continuing professional development in Texas: survey of pharmacists' knowledge and attitudes: 2008. J Am Pharm Assoc. 2010 May-Jun; 50(30): 368-374.

[7] Austin Z. CPD and Revalidation: Our future is happening now. Research in Social and Administrative Pharmacy. 2013(9): 138-141. PMid:23062784 http://dx.doi.org/10.1016/j.sapharm.2012.09.002 